\title{
YouTube as a Potential Medium for Liquid Health Communication: A Study on Women's Consciousness Regarding Breast Cancer in Bangladesh
}

Monira Begum

Assistant Professor, Dept. of Mass Communication and Journalism, University of Barishal, Bangladesh.

\begin{abstract}
This study deals with YouTube as the most influencing source and a strong tool replacement of traditional media through the uploading and providing a vast number of health contents especially on Breast Cancer (BCa) for promoting health communication throughout the world. The study has been conducted with some specific objectives, including finding out the nature and qualities of these video contents on BCa, women's involvement in searching these contents, and the sufficiency and effectiveness of these videos and content in creating awareness among women in Bangladesh. All the objectives are scrutinized in this study carefully. Moreover, the study observes that most women consider breast cancer as a non-communicable disease, which leads to a tougher situation for women taking treatment further. Importantly, this study finds a great correlation between what women want and what YouTube videos contents offer on this issue. Results of this study also show that most of the videos are enough to fulfill the respondents' expectations, while these videos cover specialist advice including on treatment, symptoms, and causes of BCa mainly. A large proportion of the percentage reveals an enormous involvement of women searching video content on BCa with some activities, like viewing and commenting in the comment section. Most of the respondents of this study agreed that all the video contents are trustable, and hoping that YouTube will be a good doctoral site for women. From the positive aspects of the respondents in answering that this study proves the effectiveness of YouTube contents on BCa in creating awareness not only among women but also mass people, and thus makes a liquid health communication between mass media and mass people.
\end{abstract}

KEYWORDS: Breast cancer (BCa), YouTube, Health communication, Mass media

\section{INTRODUCTION}

Breast cancer (BCa) is the most common type of cancer for women and now is increasing all over the world. According to the data of 2020, almost 13,028 women have been affected and 6,783 women embraced death for BCa in Bangladesh (Globocan, 2020). This is also a very common scene in Bangladesh that BCa has been termed as a non-communicable disease among people. As it is commonly thought that BCa is treated only as a female disease but most male people have no knowledge of BCa that can be a disease for males too. In Bangladesh, people use the internet to stay connected with social networking sites (SNS) for instant communication. Moreover, people want to stay connected with the cheap and lucrative information in social media. But most of the people in our country don't show any interest in health information. Sometimes, people are somehow likely to be active in searching for any health problem only on ongoing issues in the country. In this study, it is shown and discussed that women who have easy internet access can avail the benefits of YouTube videos by clicking on the contents at any time. Apart from the obtaining lots of information from YouTube, women can be aware of BCa specifically through making discussions among others women who use social media or through face-to-face communication. Besides online media, print and electronic media also upload their own contents including health-related news or videos for viewers and audiences on YouTube. People, who missed the program or news, can go and click on the YouTube link to get instant access to those uploaded programs or videos. The study finds that as a non-communicable disease, young people especially women show their interest in searching for any kind of information related BCa through YouTube.

According to January 2021, there are 4.66 billion active internet users worldwide, among them 4.20 billion people use social media actively (Johnson, 2021). And the number of YouTube viewers worldwide is about 1.86 billion (Tankovska, 2021). People in Bangladesh now use YouTube as the second popular SNS mostly. As a result, YouTube can be the most 
resourceful SNS among people especially for women in the context of BCa.

\section{Background of the Study}

According to the data of the American Institute for cancer research, $\mathrm{BCa}$ is the second most common cancer worldwide.
There are almost 22,61,419 women are affected by BCa in 2020 (Globocan, 2020). Bangladesh is facing the same challenging situation now. As for developing countries, the number of BCa affected people and deaths have been increasing more because of not having proper education and treatment in time.

\section{Number of new cases in 2020 , females, all ages}

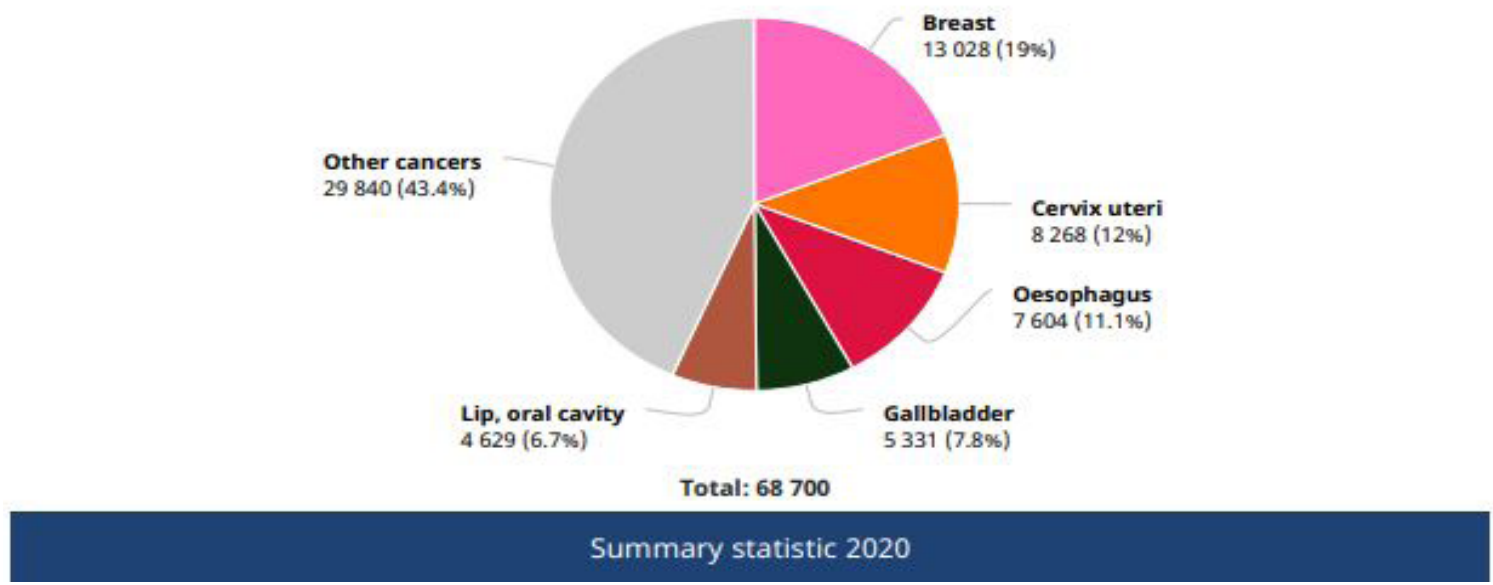

Source: Globocan, Global Cancer Observatory-Bangladesh, 2020

Age is more important for affecting BCa. A study of Nessa et al. (2018) deals about the age distribution pattern of female BCa patients in Bangladesh. Researchers reveal that from 3280 years female; get affected with this disease comparatively than western countries. In this study, researchers also observe that female people are going through this disease at an early age (Nessa et al., 2018).

Islam et al. (2016) conducted a study on women's awareness of breast screening uptake in Bangladesh in their study. Researchers found that among 1,590 aged women, 40.2\% had no formal education from 30 years to 59 in 7 districts. In this study, questions about breast cancer reveal that $81.9 \%$ of the women have ever heard of BCa, among them, $64.2 \%$ have ever heard of a BCa screening method but few of them ever performed the test. Researchers also found that there were less knowledge of rural women concerning about BCa, among them whose ages of 40-59 years old, were more likely to be aware of BCa compared 30-39 years aged people. And women, who were overweight or obese, seemed more likely to be aware of BCa compared with women who had normal body mass index (BMI). And also Women who had three or more children, were less likely to be aware of BCa compared with women who had fewer than three children. Lastly, Researchers pointed out some reasons of barriers for not undergoing screening test includes that women found no symptoms (92\%) and that they didn't know if the screening test was needed (40\%), Some women showed modesty issues $(4.7 \%)$ and religious issues $(1.7 \%)$ as other barriers (Islam et al., 2016, p. 69-73). However, after analyzing all of the aspects of different researches, it is clearly observed that there's demand of a new research in this field.

\section{Objectives of the Study}

The main objectives of this study are specified below succinctly;

1. To find the nature and quality of Bengali and Bangladeshi YouTube video contents on breast cancer spanning from 2010 to 2020

2. To find the involvement of women and their activities in You Tube in searching the contents on breast cancer, and

3. To know whether the information in those videos contents is sufficient for women or not and to the extent, if this kind of media (YouTube) paves the way for liquid health communication in Bangladesh.

\section{Rationale of the Study}

Breast cancer is increasing tension in society gradually, especially in health communication. Women feel reserved in discussing this disease publicly. In this context, YouTube video content on BCa can be a powerful tool of communication to reach general people. And for this, it is needed to evaluate women's involvement and their activities in searching video content on BCa in YouTube. As the health of the citizens is an integral part of national development, the study claims its relevance to the same.

\section{REVIEW OF LITERATURE}

In recent years, breast cancer has become one of the most leading diseases in Bangladesh, especially for women. Women from both rural and urban areas have less access to the internet; there is a barrier to their knowledge about breast cancer. Women have limited or no introduction to 
breast cancer and its campaigning through media or any participatory program organized by any public or private body. For this lack of consciousness, they suffer from this disease and are often reluctant to share the problems with others. As they feel reserved to know about it from the direct conversation, YouTube may be the most helpful media where they can learn by themselves. But here lies the same problem that breast cancer campaigning cannot reach people, especially to the women.

Due to the alarming rise of breast cancer, researchers have given concentration to it. In Bangladesh, several kinds of research have been performed, some by academicians while others by NGOs. But most of them are relating to finding out awareness among women or girls and also to overcoming barriers articles. Few articles and researches are on these fields.

Islam et al. (2019) showed non-communicable diseases as a challenge for the low and middle-income country where social media (Facebook, YouTube, Instagram, Twitter, LinkedIn, discussion forums, blogs, and microblogs and message or chat application, Facebook Messenger, WhatsApp, QQ, WeChat, Skype, Viber) could lead to a powerful tool for prevention by reaching to large population. Though having some risks and challenges of conveying incorrect information, lack of data confidentiality, monitoring, and regulation, commercial interests, equity of access, and lack of standards, a regulatory guidelines and standards needed to be developed to help avoid adverse consequences to improving health communication as well. Researchers also remarked that 'Social media also offers low-cost opportunities for awareness building via disseminating health information and campaigns to a large population and great potential to influence knowledge, attitudes, and behaviors of people for the prevention and management of Non-communicable diseases in low and middle-income countries' (Islam et al., 2019, p. 97-99).

Purabi (2019) deals with the importance of digital technology as a potential tool in health care in her study. She managed to select two social platforms (YouTube content and Facebook page mentioned) in study. She shows that young people aged 18 to 34 are likely to be connected with the social platform but the women who are poor in number; most of them watch YouTube contents than Facebook for getting any health information. Moreover, the researcher keeps her hope in the conclusion that if there's the opportunity to engage more people with digital technology lowering the internet price, SDG goals could be achieved easily (Purabi, 2019, p. 203206).

Ruppert et al. (2017) showed YouTube as a powerful source of health information in their study. They analyzed the contents of 281 YouTube videos related to sun protection and skin cancer prevention in 6 languages. Researchers also used a four-sectioned checklist to assess general information, expert-driven measures, popularity, and heuristic-driven measures. After observing the result is that the general quality of YouTube contributions is often inferior and does not deliver sustainable information. In addition, Researchers suggested in the study that 'today's respected and successful YouTube contributions are generally of superior quality, but they require professional planning, end up in costly production, and employ various additional accompanying marketing measures to make a strong impression and impact' (Ruppert et al., 2017, p. 9).

Hasamnis \& Patil (2019) suggest YouTube as a powerful health communication tool in their writing saying that 'Educators may decide to screen and choose the best educational material available online and recommend it to students for further learning. Online YouTube content with high engagement time by viewers may act as a starting filter in this process[...].This is a mammoth task, and as educators, we should make our students aware of this if they find YouTube as a medium of learning easy and comfortable'. And as medical students has to practice and heal in the real world, so this is very important to ensure 'Meaningful, appropriate, and adequate use of virtual and digital platforms can only aid in learning the theory of medicine in the early years of medical career' (Hasamnis \& Patil, 2019, p. 241).

Chandan et al. (2018) stated that Social media platform played a very powerful way to engage a greater number of people through providing lots of information regarding specific cancer prevention and supportive information for cancer survivors. After finding the result of this study Researchers agreed that face book, YouTube, Twitter user can be a great source of spreading information on various aspects of cancer prevention through a comprehensive social media campaign because of their high penetration power (Chandan et al., 2018).

Bottorff et al. (2014) conducted research aiming to develop youth-informed, gender-specific YouTube-style videos with a combination of moving text, novel images, animations, and youth-friendly music designed to raise awareness who smoke to understand how their smoking puts their female peers at risk for breast cancer and to assess youths' responses to the videos and their potential for inclusion on social media platforms. In interpreting the findings stand the inclusion of given videos in YouTube opined by participants whose ages are 11 to 19 and they are more likely to share the videos with their friends and family members with some improvements of videos. Almost all the participants strongly agree to tobacco exposure is a modifiable risk factor for breast cancer. Researchers also paid more importance to use the social platform of young people, in conclusion, stated that 'Interactive technologies hold promise for cost-effective, gender-specific messages. Not only do these media enable broad reach, but they also bring an inter-active nature to cancer prevention programs for young people' (Bottorff et al., 2014, p.166). 
Similarly, Burton et al. (2012) worked on the importance of YouTube videos in building public health community. They got the results after analyzing some anti-smoking videos, authors, subscribers, and comments that videos are not only about smoking behavior but also focus on humorous or sexual content too. Researchers also showed YouTube as a powerful tool in making an author-friend community, a commenter community to perceive data and gauge the feeling, perception, and reaction of the public to the messages that are presented in YouTube. (Burton et al., 2012, p.3-8)

Jacobsen \& Jacobsen (2011) discussed the campaigning impactand comparative results in their research. In this study, they have evaluated the effect of one of the best known and longest-running detection campaigns, the National Breast Cancer Awareness Month (NBCAM). They have compared the campaigning impacts and found rapid advancement of breast cancer advocacy before the '80s, the initial period of the campaign. They further show the factors working behind such changes (Jacobsen, Jacobsen, 2011).

\section{Theoretical Framework}

Media Richness Theory (MRT) was introduced by Daft and Lengel in 1986 as an extension of information processing theory. Daft and Lengel (1986) proposed that technologybased channels of information are rich text sources than the other mediums. 'Media richness theory claims that the effect is better when communicator use richer media' (Dennis \& Kinney, 1998) and richness means the ability of the medium to transmit the information from sender to receiver. It can be described as; something's written by someone can not attract more people than videos because this shows the gestures and expressions of the other person while saying those words. Media richness theory is about richness in communication and that the communication process should involve a rich source for effective communication.

As the communication process goes through varieties framing techniques and ways that is why the source of information should be rich. This theory has its richness having of the capacity for immediate feedback, the number of cues and channels available, holding a variety of language and the degree to which the receiver can focus (Daft \& Lengel, 1986). According to this theory, YouTube is media that is full of information with unlimited videos having so many languages, has instant feedback option through the comments and video contents on BCa that shows its richness. The viewers can easily understand the message in the video with the help of the visuals with the voice-over. It contains thousands of tutorial videos in almost every language for its audience. Moreover, this study demands this theory for its relevance to finding out the objectives of this study.

\section{METHODOLOGY}

The study follows a quantitative approach. As breast cancer is an issue of global and national significance, the research is designed carefully so that necessary information can be collected efficiently from different authorized sources. The study is, however, deals with content analysis of the YouTube videos on Breast Cancer. Total numbers of 50 YouTube video contents have been observed using random sampling for this study for observing and analyzing the adequacy and qualities of these videos.

In order to yield the specific objectives of this study, also a survey has been conducted on young women. In total number of 100 women, ages 18-30 years, have been selected using purposive sampling on the basis of their known engagement in the topic in questions but only 50 women joined the survey and the rest felt uneasy in this regard and rejected joining the survey. That brings the limitation for the study.

\section{DATA ANALYSIS AND RESULTS DISCUSSION}

\section{The list of YouTube video contents on BCa spanning from 2010 to 2020}

Data of the below list shows that 31 (62\%) videos have a thousand viewers while $4(10 \%)$ videos have a million viewers. This shows total 35 (70\%) videos have a high number of people's engagement with YouTube including their valuable comments. But the less percentage resembles of low engagement of people where only 15 videos have less than a thousand viewers. In the list, almost all videos uploader are from health-related sites, TV report based and specialists who mainly focus on breast cancer causes, symptoms, screening, prevention and others. 6 videos have been observed from 2020 where 4 videos get thousand people's engagement, 14 videos have been observed from 2019 where 9 videos have thousand engagement and 2 videos have million engagement of people, 20 videos have been observed from 2018 where 13 videos have thousand engagement and 1 video has million engagement of people, 3 videos have been observed from 2017 where 1 video has thousand engagement, 4 videos have been observed from 2016 where 2 videos have a thousand viewers while 1 video has million engagement of people, 1 video has been selected from each year from 2015, 2013 and 2012 where 2 videos of 2013 and 2012 have thousand number of people engagement.

The other thing is that the contents on $\mathrm{BCa}$ are getting priority in YouTube from the beginning of the year 2020 but unfortunately got stopped uploading for the possible reasons of the breakdown of corona in Bangladesh after March. Videos in 2019 also cover most of the months of the year not only giving the priority in BCa awareness month. In 2018 , most of the videos are seen uploaded focusing around the BCa awareness month and after while less focusing on other months, and also the same scenario in 2017. Almost the same results from the year 2016, 2015, 2013, and 2012 show that videos are uploaded around the BCa awareness month not covering the every months of these years. 
YouTube as a Potential Medium for Liquid Health Communication: A Study on Women's Consciousness Regarding Breast Cancer in Bangladesh

\begin{tabular}{|c|c|c|c|}
\hline Serial & Content creator or publisher & \begin{tabular}{|l|} 
Viewers \\
\end{tabular} & Date of publishing \\
\hline 1 & DR Ghosh presents & 1,544 & Jan, 2020 \\
\hline 2 & Doctorola TV & 1,639 & Jan, 2020 \\
\hline 3 & RAJ TV & 23 & Jan, 2020 \\
\hline 4 & Health care Bangla & 16,301 & Feb, 2020 \\
\hline 5 & Rotary club of Dhaka Golden city & 15 & Feb, 2020 \\
\hline 6 & Bongo BD & 3,919 & Mar, 2020 \\
\hline 7 & Sustho protidin & $58,22,017$ & Jan, 2019 \\
\hline 8 & Doctors Tv BD & 2,253 & Jan, 2019 \\
\hline 9 & Heal life & $5,05,628$ & Jun, 2019 \\
\hline 10 & Health cafe & 8,748 & Jun, 2019 \\
\hline 11 & Health care Bangla & 15,530 & Jul, 2019 \\
\hline 12 & All time News & 2,586 & Aug, 2019 \\
\hline 13 & Health care Bangla & 2,343 & Sep, 2019 \\
\hline 14 & Roche & 1,357 & Sep, 2019 \\
\hline 15 & All infinite everything you want & 473 & Sep, 2019 \\
\hline 16 & BanglaVision program & 3,572 & Oct, 2019 \\
\hline 17 & BBC News Bangla & 1,942 & Oct, 2019 \\
\hline 18 & U S Embassy Dhaka & 328 & Nov, 2019 \\
\hline 19 & Maasranga program & 48,327 & Nov, 2019 \\
\hline 20 & Take care Bangla & 334 & Dec, 2019 \\
\hline 21 & Somoy Tv & 1,555 & Jan, 2018 \\
\hline 22 & channel24 YouTube & 1,960 & Jan, 2018 \\
\hline 23 & MyTv Bangladesh & 946 & Feb, 2018 \\
\hline 24 & Doctorola TV & 62,676 & Jul, 2018 \\
\hline 25 & Medical science Lesson & 54,844 & Jul, 2018 \\
\hline 26 & Prof. Dr. Salma Yesmin Choudhry & 40,268 & Jul, 2018 \\
\hline 27 & Somoy Tv & 409 & Jul, 2018 \\
\hline 28 & Maasranga program & 9,540 & Sept, 2018 \\
\hline 29 & Habibullah Talukder & 37 & Sep, 2018 \\
\hline 30 & Channel 69 & 36,641 & Sep, 2018 \\
\hline 31 & Sastho somossa somadhan & 438 & Oct, 2018 \\
\hline 32 & Maasranga program & 18,914 & Oct, 2018 \\
\hline 33 & Shanti cancer foundation & 39 & Oct, 2018 \\
\hline 34 & Doctorology & 26,086 & Oct, 2018 \\
\hline 35 & RTV lifestyle & $1,32,306$ & Oct, 2018 \\
\hline 36 & Dr Ferdous USA & 5,007 & Dec, 2018 \\
\hline 37 & Doctors Tv BD & 34,367 & Dec, 2018 \\
\hline 38 & All the times & 994 & Dec, 2018 \\
\hline 39 & IARC WHO & 11,867 & Dec, 2018 \\
\hline 40 & Dr Ferdous USA & 18,341 & Dec, 2018 \\
\hline 41 & Digi Bangla TV & 953 & May, 2017 \\
\hline 42 & Doctorola TV & 87,458 & Oct, 2017 \\
\hline 43 & Channel 24 & 474 & Oct, 2017 \\
\hline 44 & Doctors Tv BD & 2,106 & Mar, 2016 \\
\hline 45 & Health care Bangla & $2,70,829$ & Mar, 2016 \\
\hline 46 & Health care Bangla & 19,169 & May, 2016 \\
\hline
\end{tabular}


YouTube as a Potential Medium for Liquid Health Communication: A Study on Women's Consciousness Regarding Breast Cancer in Bangladesh

\begin{tabular}{|l|l|l|l|}
\hline 47 & Cancer Awareness foundation of Bangladesh & 407 & Oct, 2016 \\
\hline 48 & Habibullah Talukder & 60 & Nov, 2015 \\
\hline 49 & Habibullah Talukder & 7,326 & Oct, 2013 \\
\hline 50 & Grand Challenges Canada & 1,937 & May, 2012 \\
\hline
\end{tabular}

Source: Data collected from YouTube on 28/08/2020

\section{Narrators and vocalists of the video contents on BCa}

The selected video contents have been observed carefully where most of the narrators and vocalists of $54 \%$ are male while $40 \%$ are female. The rest videos of $6 \%$ have the absence of vocalists only having music and still pictures.

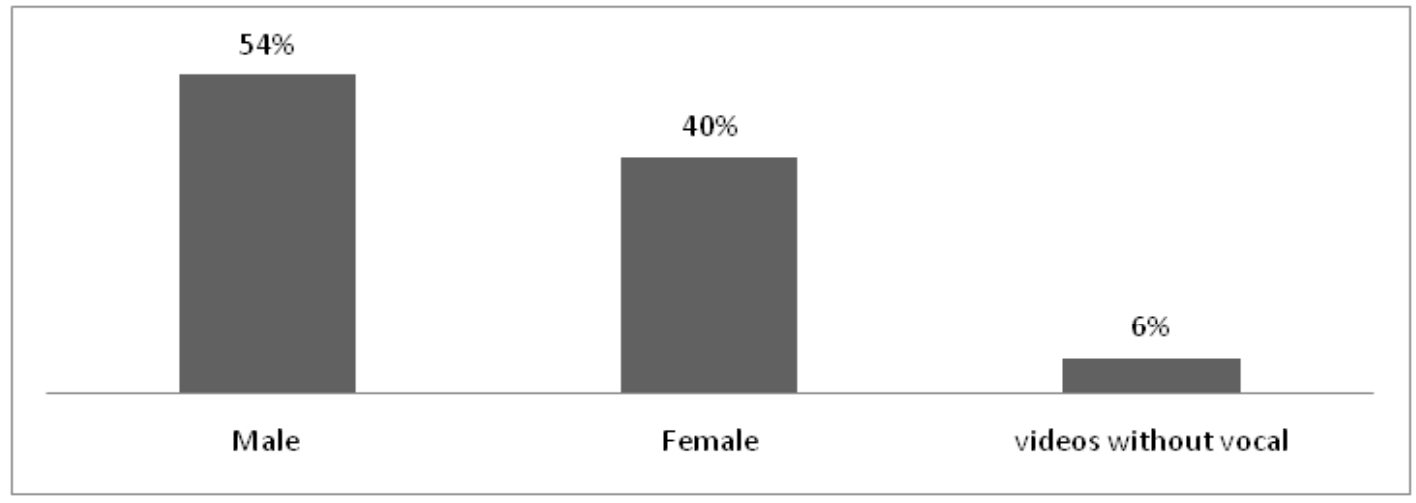

\section{Types of YouTube video contents on BCa}

The selected video contents on BCa has been analyzed and scrutinized carefully to find out the nature and types of the videos. Findings show that among all of the videos, 58\% covers prevention and treatment content, 30\% covers causes and symptoms content, $10 \%$ covers BCa screening and, only $4 \%$ covers patient's experience contents.

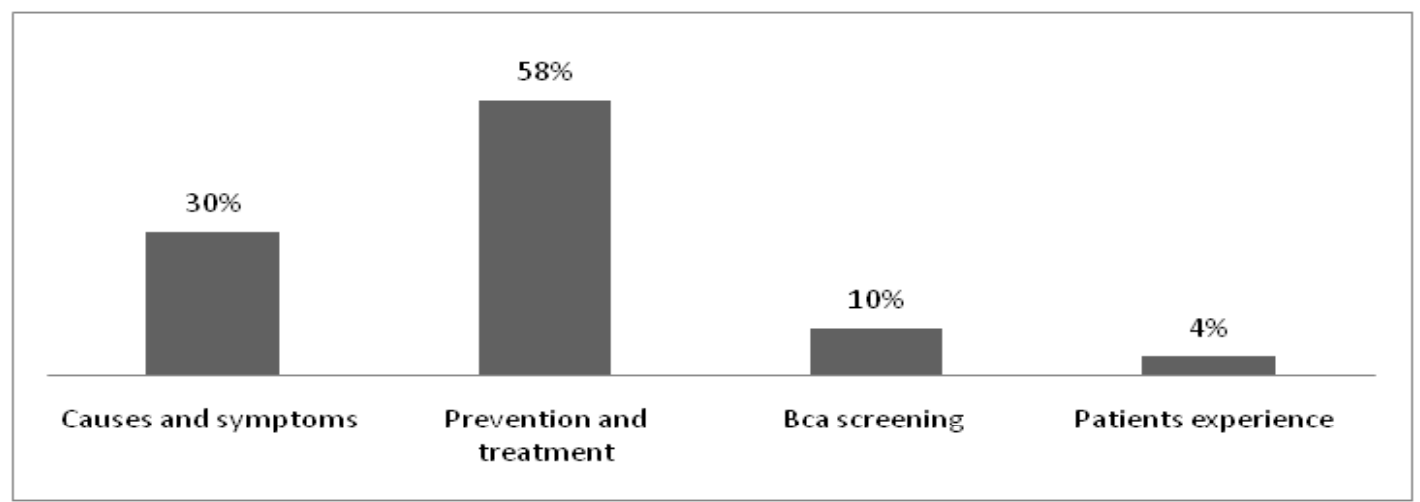

\section{Source of advice, tips, and others' information on BCa}

Findings also show that most of the YouTube videos content on $\mathrm{BCa}$ is about the advice or tips from the specialist and unknown vocalists or report-based videos. The result finds that $64 \%$ of YouTube content on BCa is about the advice from specialists, $18 \%$ covers the health tips on BCa from the unknown vocalists, and $18 \%$ is about the report-based videos.

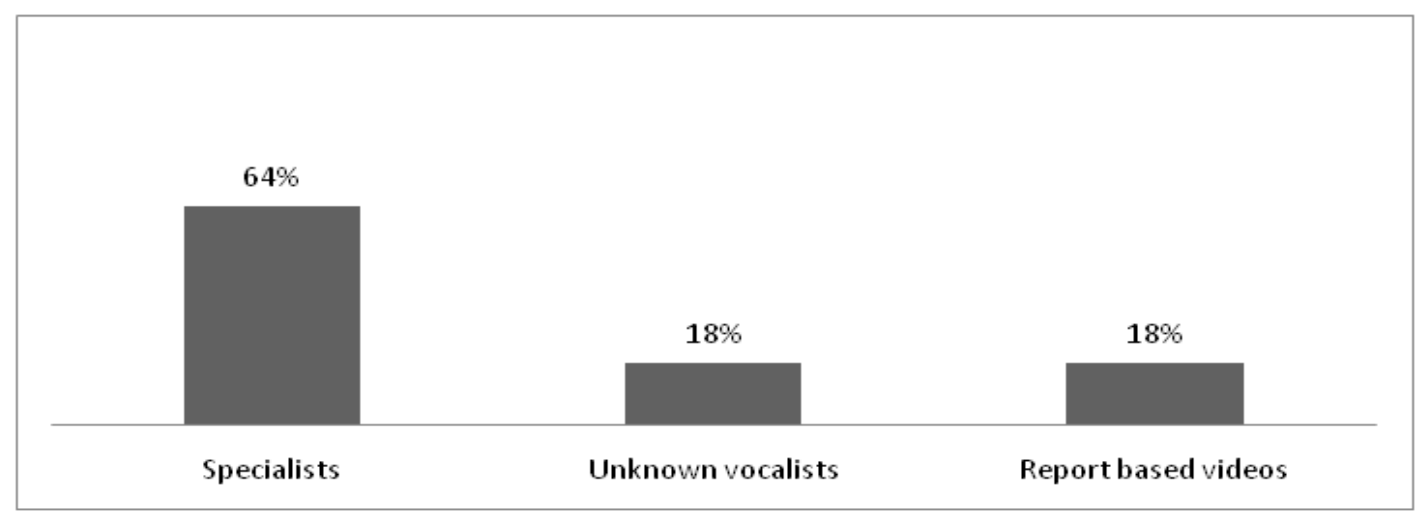




\section{SURVEY DATA ANALYSIS}

\section{Thinking about breast cancer}

Most of the respondents of this study around $60 \%$ consider breast cancer as an non-communicable disease while only the less percentage of $40 \%$ respondents think this a communicable disease.

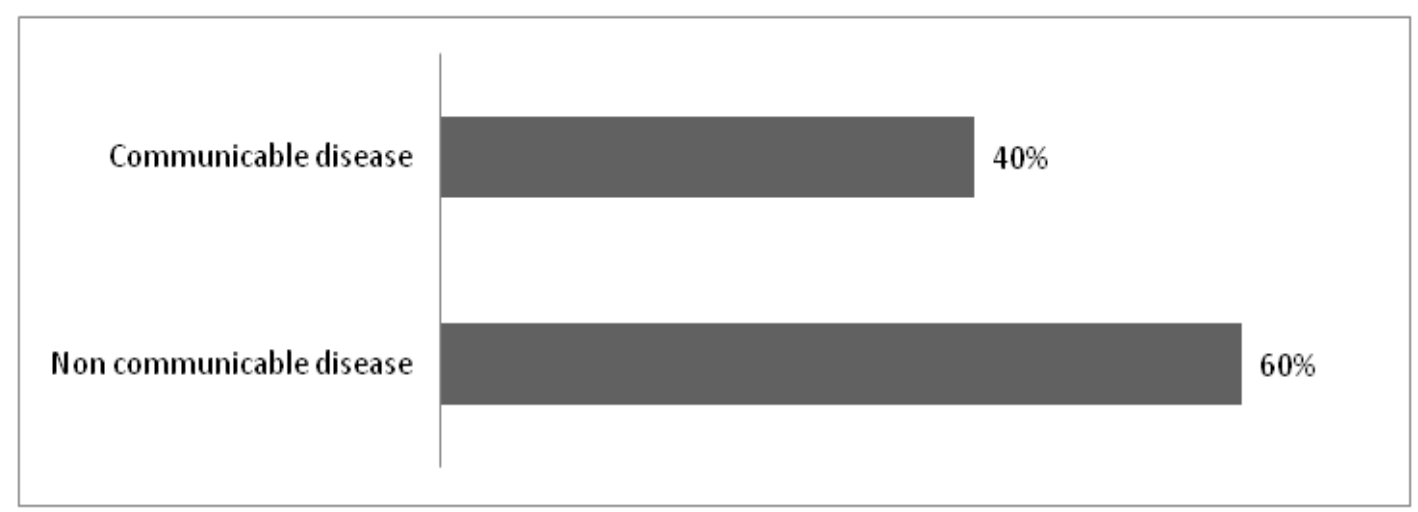

\section{From the source respondents came to know about BCa}

According to the survey results, most of the respondents of $44 \%$ get to know the information about BCa from YouTube while close percentage goes for family members $40 \%$ where respondents depend on the second most. Also $16 \%$ of respondents came to know about BCa from their friends and peer groups. 0\% percent for the 'heard the first time' shows that all respondents here are well informed about $\mathrm{BCa}$.

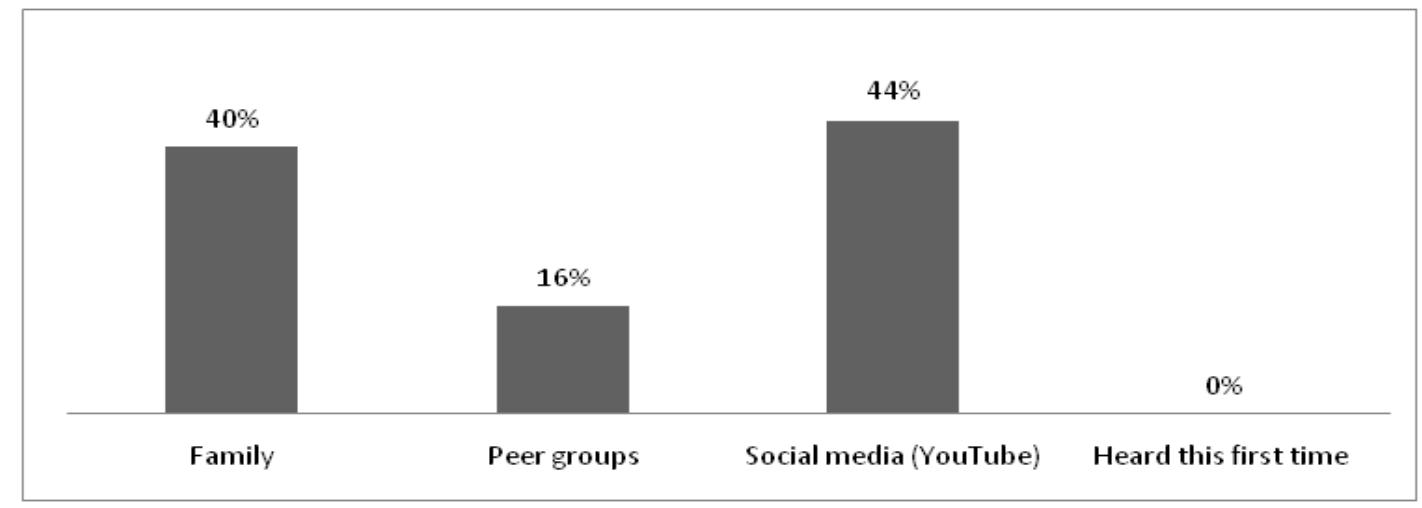

The reasons why female can be infected by Breast Cancer (BCa)

Most of the respondents of $60 \%$ of this study reveal that they don't have any idea about BCa. $12 \%$ of respondents reply that BCa can affect not feeding breast milk to the baby. 5\% of them blame for not getting married early and 3\% respondents mark the cause for having an early period while $20 \%$ of respondent shows that they believe all of the reasons here. This data shows a very poor knowledge the respondents have actually.

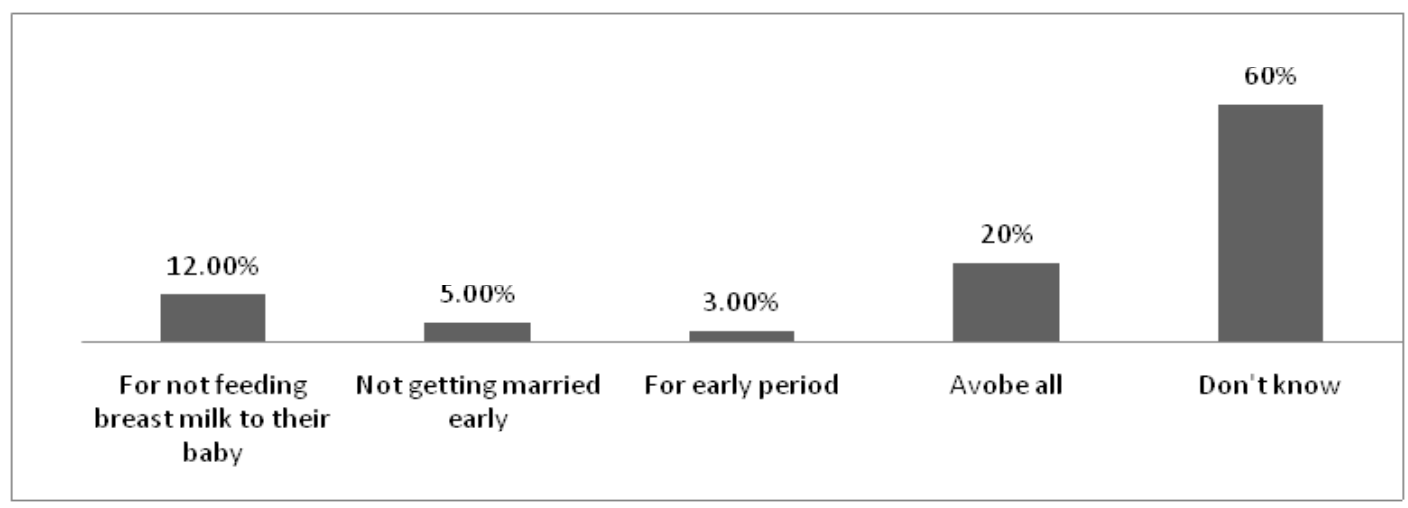

Making a free and open discussion with the male partner or male friend about BCa

As a non-communicable disease, it is normal to have negative answers in this regard. The majority, 54\% of respondents say 
that they don't feel free to discuss with their male partner or male friends about BCa while $46 \%$ of respondents agree that they feel easy to make any conversation with male partner or friends in this regard.

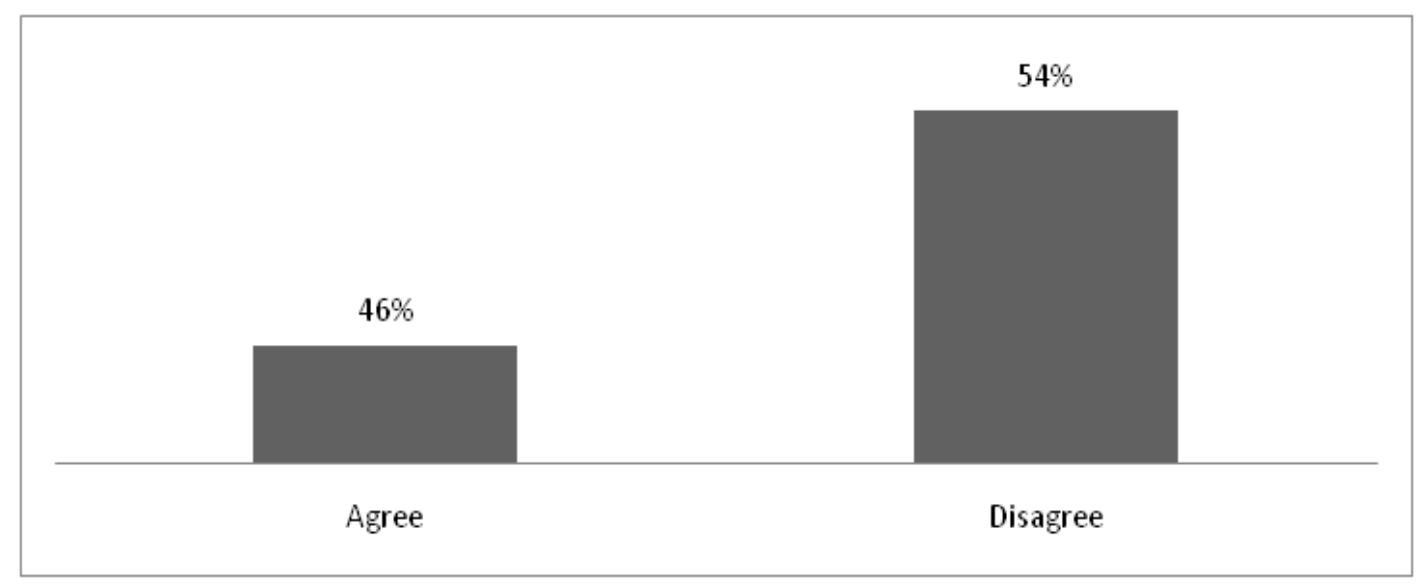

\section{YouTube as a powerful tool about BCa campaign than any other media}

Data shows that the highest percentage covers a very positive perspective here. $82 \%$ of respondents advocate that YouTube can play as a very powerful tool for BCa campaign than any other media where the less percentage of $18 \%$ of respondents doesn't agree with this statement.

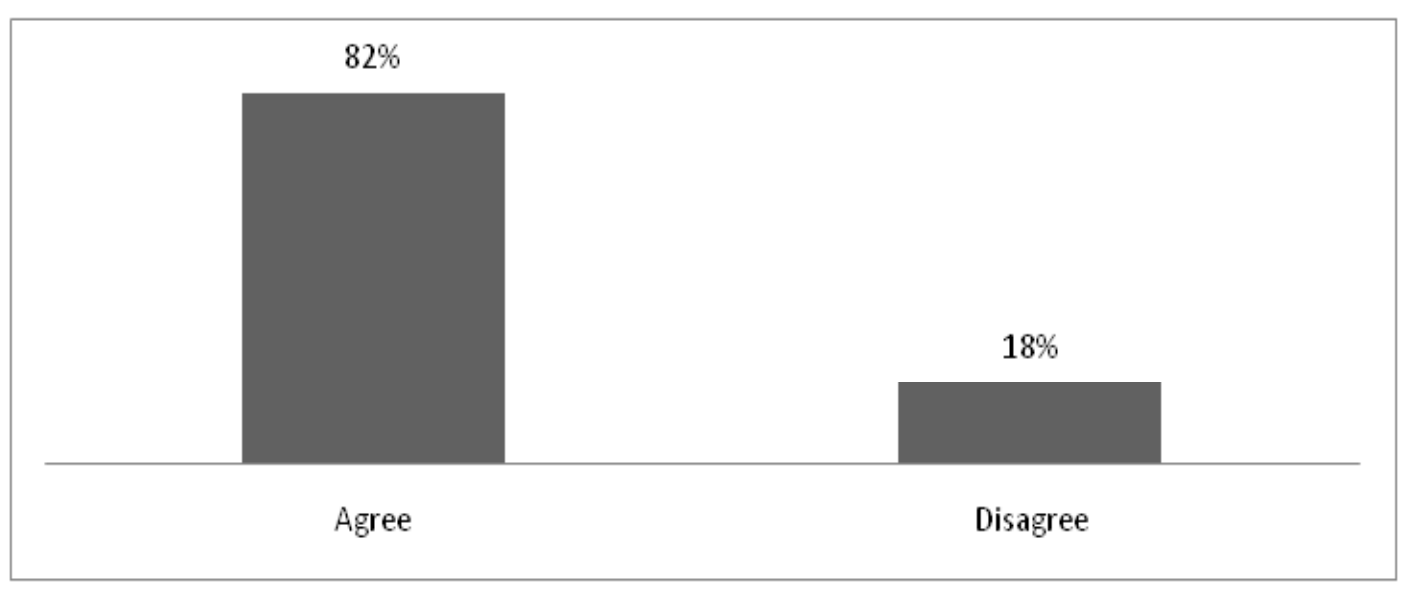

\section{Trustworthiness of the YouTube contents on BCa}

The respondents were asked about how trustworthiness the YouTube contents on BCa has. About 26\% of respondents reply that they believe all the YouTube contents on BCa fully while $66 \%$ of respondents say that some of the contents are believable and some are not. But only a few numbers of respondents of $8 \%$ don't have belief in YouTube contents on BCa.

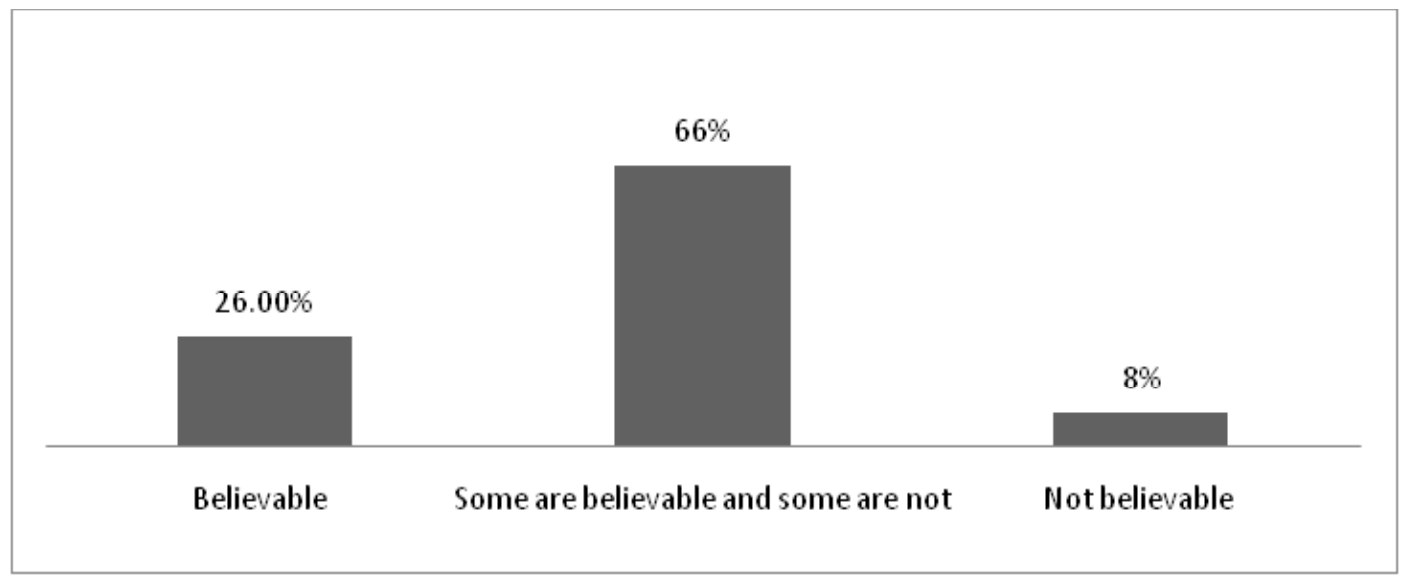

\section{Fulfilling expectation through YouTube video contents}

$60 \%$ of respondents opine that YouTube contents on BCa fulfilled their expectation but $40 \%$ of them show disappointment in this regard. 


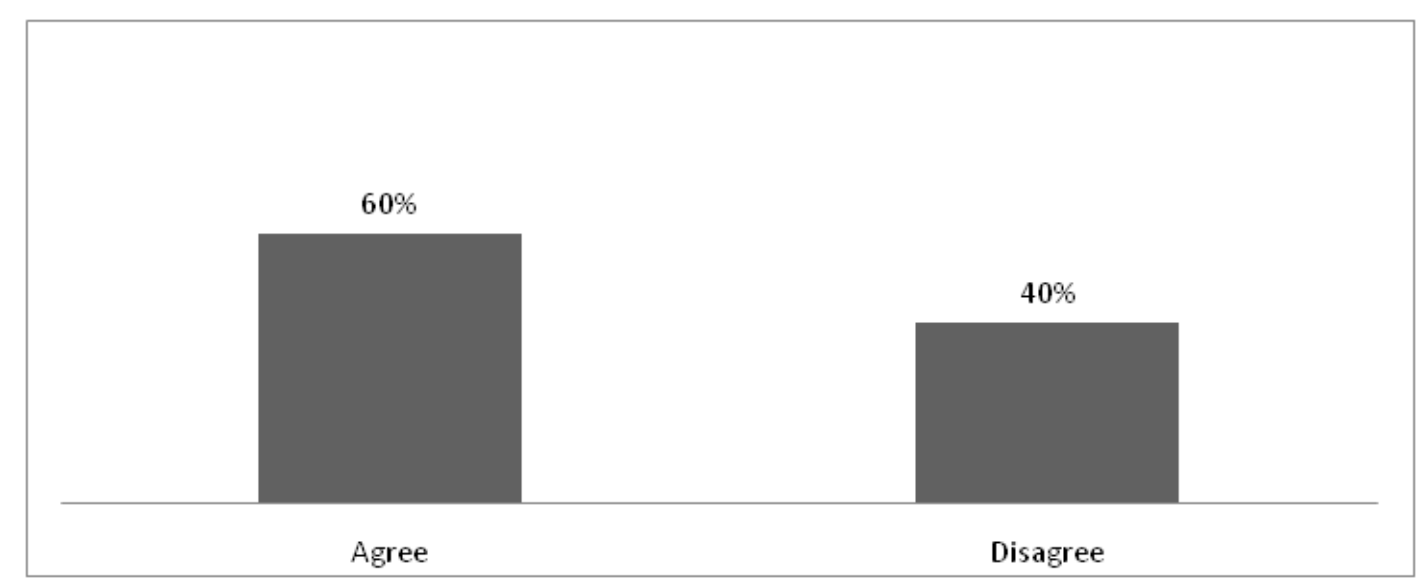

\section{YouTube as a good doctoral site for women}

Very positive opinions have been found from this question that $75 \%$ of respondents think YouTube as a good doctoral site for women while only $25 \%$ of respondents don't think so. But the respondents who think positively, among them $60 \%$ share anything of YouTube content on BCa with your other female friends/family members. Comparatively only $40 \%$ don't share but have the knowledge on it.

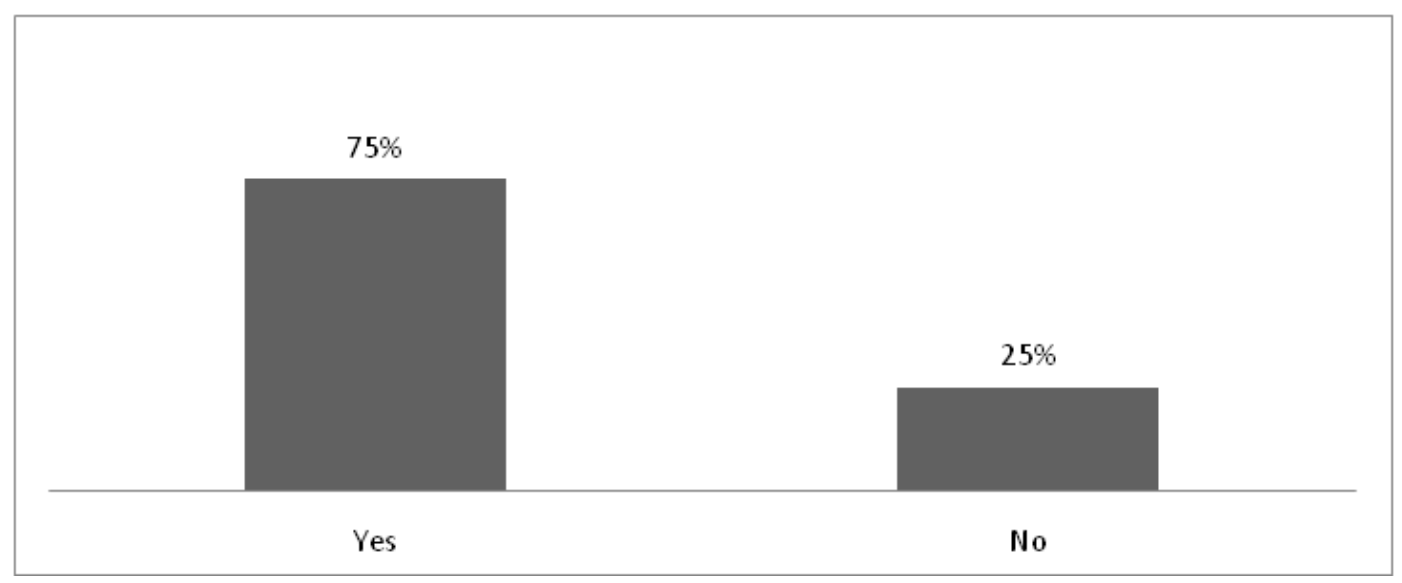

\section{Does YouTube answer the questions of BCa to viewers?}

Data shows that respondents who watch YouTube content on BCa hope for getting feedback from YouTube through asking questions. $65 \%$ of respondents reply that they YouTube gives answers the questions sometimes while $25 \%$ says that they get answer always but only $10 \%$ disagrees and says they don't think so.

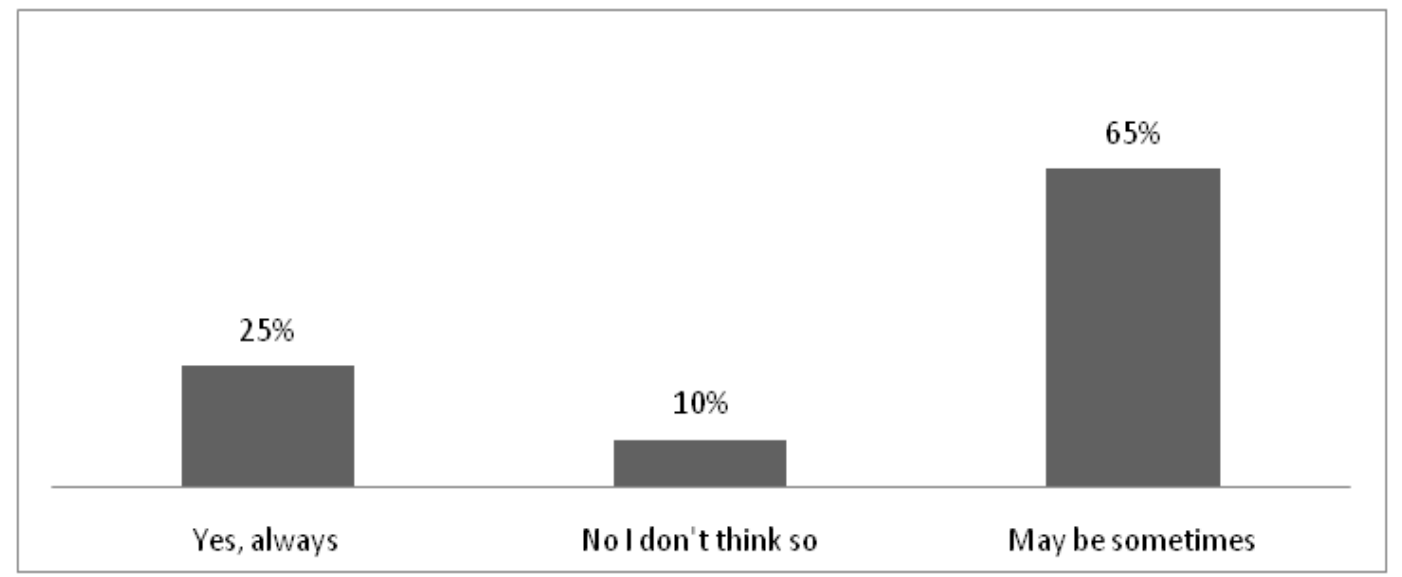

\section{The male vocalists on BCa content narration in YouTube}

Following the question about the male vocal and analysis on BCa contents narration in YouTube shows a positive notion for study. About $66 \%$ of respondents don't mind while $16 \%$ of respondents find male vocal on BCa content narration as amazing. On the other hand, $18 \%$ of respondents find these uncomfortable things in YouTube. 


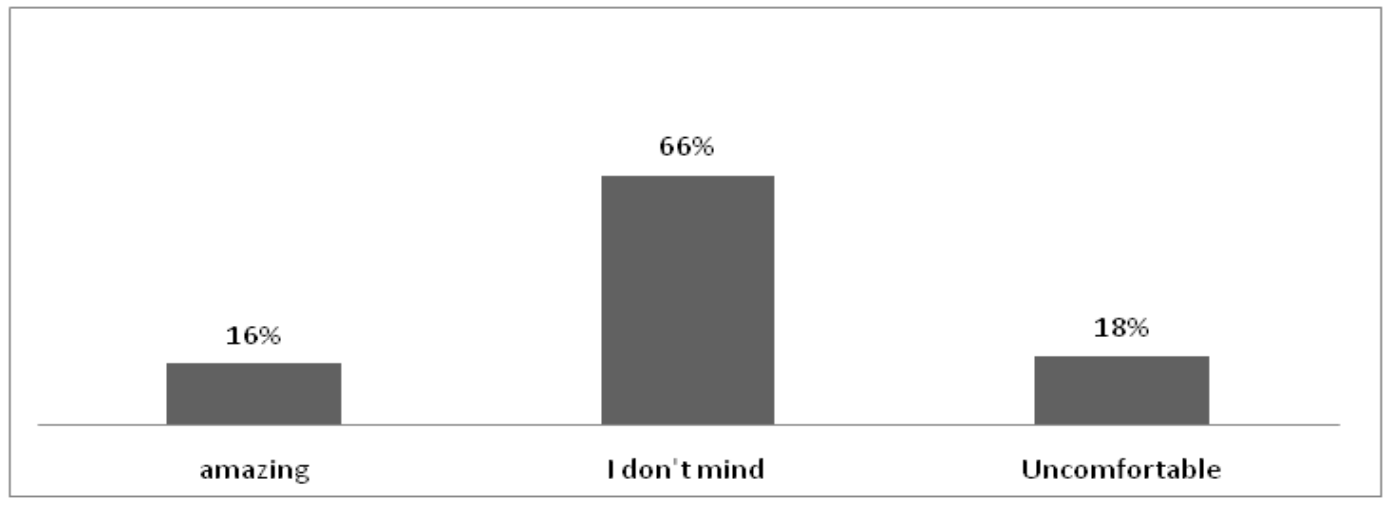

The necessary steps should YouTube do more to improve their content about BCa

Most of the respondents explain YouTube contents on BCa as a very effective way to respondents. That is why all respondents have provided some suggestions. According to the data, $65 \%$ of respondents suggest that YouTube content creator should call specialists or make the content including specialists' information more, $10 \%$ of respondents think that content creators should call patients who will explain everything from their personal experience that would be effective too. But $25 \%$ of respondents think that all information on $\mathrm{BCa}$ on YouTube is good enough and there is no need of adding anything.

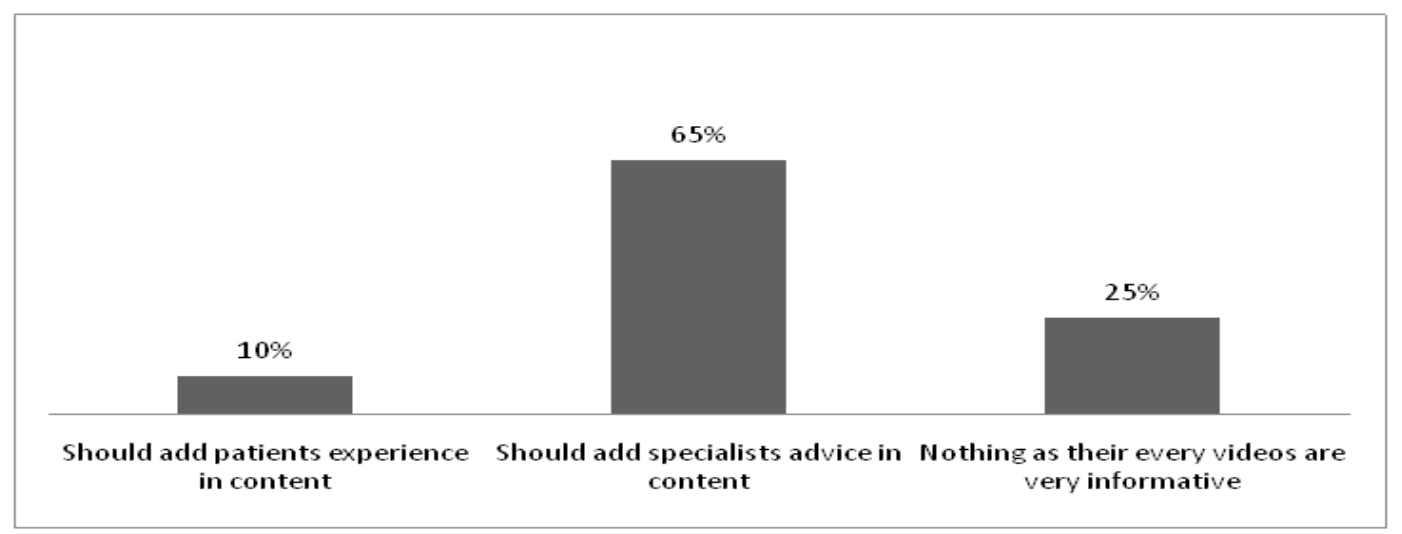

The way how YouTube contents on BCa play as powerful media in building public health community and make a liquid health communication

Respondents of this study points out some ways how YouTube contents on BCa play as powerful media in building public health community and make a liquid health communication. Data shows that the majority respondents of $56 \%$ say YouTube can provide more meaningful and adequate contents on $\mathrm{BCa}, 23 \%$ of respondents state that YouTube can ensure standards and regulatory control in content and $21 \%$ respondents show that there should be more videos on voluntary work on BCa.

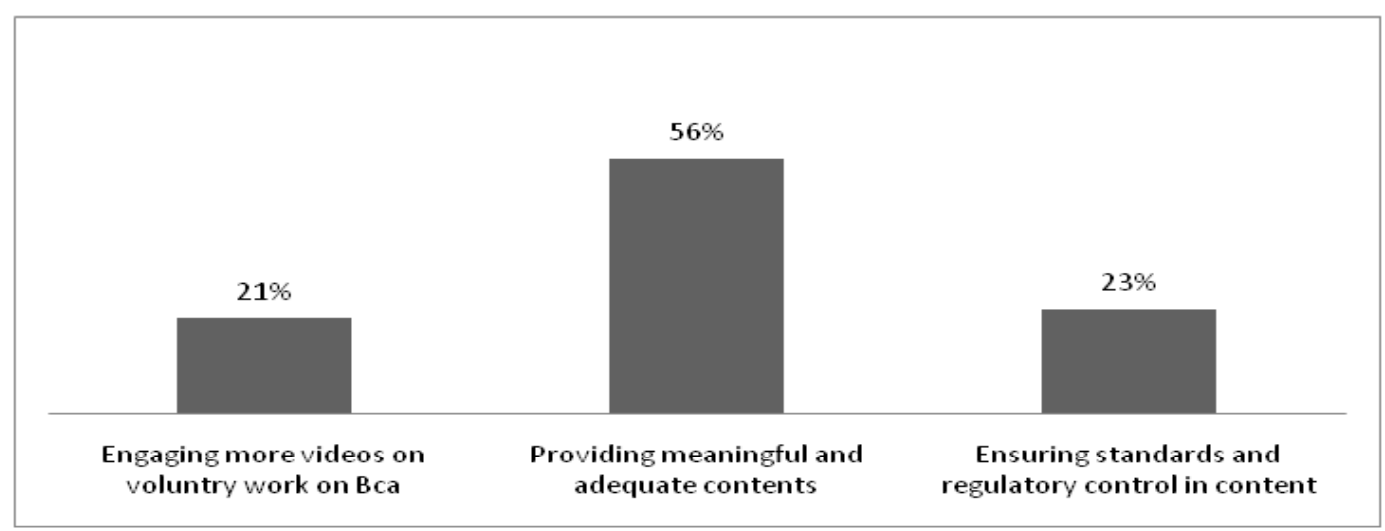

\section{Campaign/advertisement video of Nayla Nayeem in creating awareness on BCa}

Most of the respondent of this study take Nayla Nayeem's campaign/advertisement in creating awareness on BCa positively. $75 \%$ of the respondents agree that the content of Nayela nayeem's video on BCa is good making and very informative. On the other hand, $12 \%$ of respondents say that video has her full of sexual appearance while $13 \%$ of respondents mark this video as gender insensitive. 


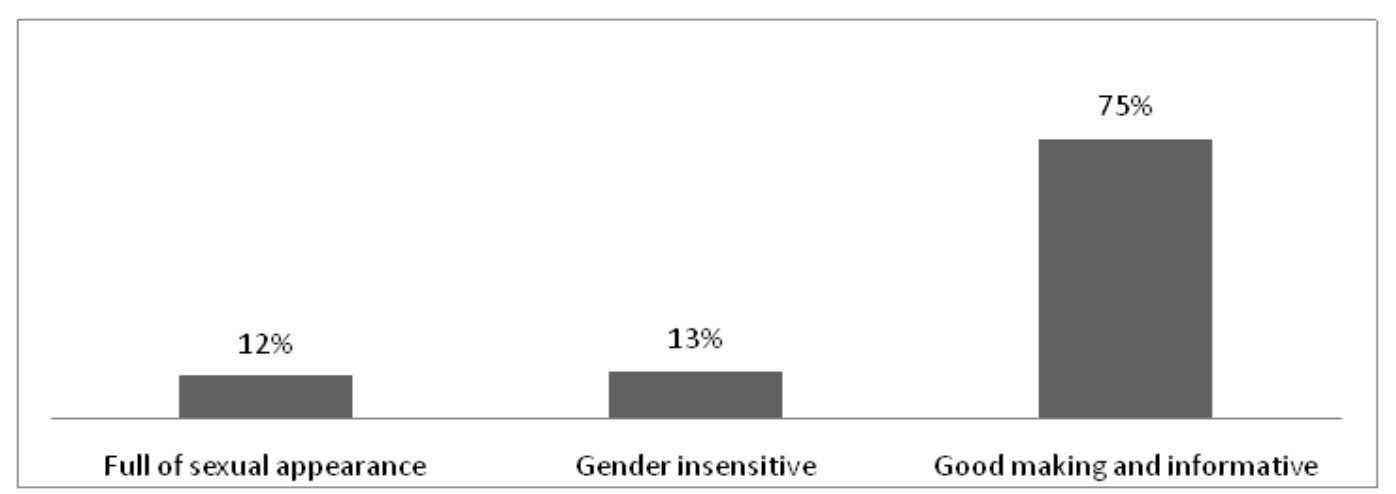

\section{RESULTS DISCUSSION}

\section{The types and quality of YouTube videos content on breast cancer spanning from 2010 to 2020}

The random selection of YouTube videos content on BCa during these 10 years shows that before 2016, most of the YouTube contents are seen to uploaded only around in BCa awareness month. After 2016 data of this study finds that most of the content of the videos covers not only awareness month but others months of the year. Among all of the videos, $58 \%$ covers prevention and treatment videos content, $30 \%$ covers causes and symptoms videos content, $10 \%$ covers BCa screening contents and, only $4 \%$ covers patients' experience contents. However, the result shows that in Bangladesh, the majority concentration goes for the discussion of prevention and treatment contents than causes, symptoms, screening, and other things in YouTube videos on BCa.

There is also a positive thing found between the analysis of video content and the results from the respondents' data about the narrators or male vocalists of the video content. From the results of content analysis, it is observed that $54 \%$ of video content narrators or vocalists are male while $40 \%$ are female. On the other hand, almost $82 \%$ of respondents of the survey don't mind having male vocals on BCa contents narration in YouTube.

Besides, among all videos, $64 \%$ of YouTube contents on $\mathrm{BCa}$ is about the advice from specialists, $18 \%$ covers the health tips on $\mathrm{BCa}$ from the unknown vocalists, and $18 \%$ is about the report-based videos. This result is very promising because of the respondents' responses. $65 \%$ of respondents suggest that YouTube content creators of uploaders should call specialists or make the content including specialists' information more. The comparison between these data suits well and has positive relation too.

There is also a question about Nayela Nayeem's BCa campaign video where $75 \%$ of the respondents opine positively and agree that the content is good making and very informative. On the other hand, $12 \%$ of respondents say that the video has her full of sexual appearance while $13 \%$ of respondents mark this video as gender insensitive. Despite these negative comments, results show very positive thinking of YouTube content on BCa recently.

\section{Involvement of women and their activity in YouTube in searching the contents on breast cancer}

As $60 \%$ of respondents consider breast cancer as a noncommunicable disease, it can be assumed that they may stay connected with others media than communicating or discussing with people. From the analysis of video content, a huge number of people's engagements are found with YouTube. This shows a total of $35(70 \%)$ videos have a high number of people's engagement with YouTube including their valuable comments. Almost all videos uploader are from health-related sites, TV report based and specialists which focused mainly on breast cancer causes, symptoms, screening, prevention, and others. The result from the respondents' response in this issue proves the peoples' more involvement with YouTube video content than other sources of media.

\section{Sufficiency of the content information of YouTube videos on BCa for women}

According to the survey results, $82 \%$ of respondents advocate that YouTube can play as a very powerful tool about $\mathrm{BCa}$ campaign than any other media. Respondents also have strong trust in YouTube video content. 26\% of respondents opined that they have full belief in all YouTube contents on BCa while $66 \%$ of respondents say that some of the contents are believable and some are not. This also shows positive results in regards to trustworthiness.

\section{Effectiveness of YouTube contents for campaigning on BCa consciousness}

Almost $60 \%$ of the respondents reveal that they don't have any idea about the causes of BCa. In spite of having a lack of knowledge about $\mathrm{BCa}, 75 \%$ of respondents think that YouTube can be a good doctoral site for them by providing enough information and content. 90\% of respondents find positive responses from YouTube in questions' answering. Respondents opine that YouTube answers their questions.

\section{YouTube as a potential tool/medium for liquid health communication}

According to the results of the survey, $60 \%$ of respondents share anything of YouTube contents on BCa with female friends and family members. It is also observed that $60 \%$ of the respondents reveal that YouTube contents on BCa fulfill 
their expectation but $40 \%$ of them show disappointment in the content. Respondents also give some suggestion to make YouTube a tool for liquid health communication. 56\% of respondents say YouTube can provide more meaningful and adequate contents on $\mathrm{BCa}, 23 \%$ of respondents state that YouTube can ensure standards and regulatory control in content and $21 \%$ of respondents show that there should be more videos on voluntary work on BCa.

\section{CONCLUSION}

The results of the study show the correlation between the results from contents data analysis and the results from the survey analysis. The study also proves that YouTube has sufficient video contents on BCa which is able to fulfill the women's expectation. Finally, this study reveals YouTube as a strong tool for everyone especially for women campaigning on BCa in creating consciousness more than other media.

\section{REFERENCES}

1. Bottorff, J. L., Struik, L.L., Bissell, Laura J.L., Grahamb, R., Stevensb ,J., Richardson, C.G.(2014). A social media approach to inform youth about breast cancer and smoking: An exploratory descriptive study. Collegian Journal of the Royal College of Nursing Australia, 21, p: $159-168$

2. Burton, S., Morris, R., Dimond, M., Hansen, J., GiraudCarrier, C., West, J., Hanson, C., Barnes, M. (2012). Public Health Community Mining in YouTube. IHI, Miami, Florida, USA.

3. Chandan, K., Yadav, A., Chandra, A., Mehrotra, R. (2018). Using Social Media as an Effective Tool for Motivating Cancer Prevention. Journal of Global Oncology, Conference Paper, 28

4. Dennis, A. R., \& Kinney, S.T. (1998). Testing Media Richness Theory in the New Media: The Effects of Cues, Feedback, and Task Equivocality. Information Systems Research, 9(3)

5. Daft, R.L. \& Lengel, R.H. (1986). Organizational information requirements, media richness and structural design. Management Science, 32(5), p: 554-571.

6. Globocan (2020), Estimated number of new cases in 2020, worldwide, female, all ages. Global Cancer Observatory, WHO https://gco.iarc.fr/today/onlineanalysis-pie

7. Globocan (2020). Number of new cases in 2020, both sexes, all ages. Global Cancer Observatory -Bangladesh, WHO https://gco.iarc.fr/today/data/factsheets/ populations/50-bangladesh-fact-sheets.pdf

8. Hasamnis, Ameya., Patil, Sapna. (2019). YouTube as a tool for health education, Journal of Education and Health Promotion, 8 (241)

9. International Agency for Research on Cancer, WHO, 2018 (reserved in The Global Cancer Observatory, 2019)

10. Islam, S.M.S., Tabassum, R, Liu, Y., Chen, S., Redfern, J., Kim, S., Ball, K., Maddison, R., Clara, K. C. b. (2019). The role of social media in preventing and managing noncommunicable diseases in low-and-middle income countries: Hope or hype?, Health Policy and Technology, (8) p: 96-101

11. Islam, R.M., Bell, R.J., Billah, B., Hossain, M.B., Davis, S.R. (2016). Awareness of breast cancer and barriers to breast screening uptake in Bangladesh: A population based survey. ELSEVIER, Maturitas (84), p: 68-74

12. Johnson, J (2021). Worldwide digital population as of January 2021, Statista https://www.statista.com/ statistics/617136/digital-population-worldwide/

13. Jacobsen, G.D., Jacobsen, K.H. (2011). Health Awareness Campaigns and Diagnosis Rates: Evidence from National Breast Cancer Awareness Month. Journal of Health Economics, (30), p: 55-61

14. Nessa,A., Hussain, T., Alam, S.M., Faruk,I., Jahan, I. (2018). Age distribution pattern of female breast cancer patients in Bangladesh developing early and presenting late. International Surgery Journal, 5(2), p: 379-382

15. Purabi, Dr. N.S. (2019). Digital revolution in healthcare: Potential tool for achieving health equity in Bangladesh. International Journal of Human and Health Sciences, 03 (4)

16. Ruppert, L., Køster, B., Siegert, A.M., Cop, C., Boyers, L., Karimkhani,C., Winston, H., Mounessa, J., Dellavalle, R.P., Reinau, D., Diepgen, T., Surber, C. (2017). YouTube as a source of health information: Analysis of sun protection and skin cancer prevention related issues. Dermatology online journal, 23(1)

17. Tankovska, H. (2021). Global number of YouTube viewers 2016-2021. Statista https://www.statista. com/statistics/805656/number-youtube-viewersworldwide/

Citation: Monira Begum, "YouTube as a Potential Medium for Liquid Health Communication: A Study on Women's Consciousness Regarding Breast Cancer in Bangladesh", American Research Journal of Humanities and Social sciences, Vol 8, no. 1, 2022, pp. 14-25.

Copyright (C) 2022 Monira Begum, This is an open access article distributed under the Creative Commons Attribution License, which permits unrestricted use, distribution, and reproduction in any medium, provided the original work is properly cited. 